\title{
LINKING DIVIDENDS AND CAPITAL INJECTIONS - A PROBABILISTIC APPROACH
}

\author{
HANSJÖRG ALBRECHER AND JEVGENIJS IVANOVS
}

\begin{abstract}
In the context of collective risk theory, we give a sample path identity relating capital injections in the original model and dividend payments in the time-reversed counterpart. We exploit this duality to provide an alternative view on some of the known results on the expected discounted capital injections and dividend payments for risk models driven by spectrally-negative Lévy processes. Furthermore, we present a probabilistic analysis and simple resulting expressions for a model with two dividend barriers, which was recently shown by Schmidli [10 to be optimal in various Lévy risk models when maximizing the difference of dividend payments and injections in the presence of tax exemptions.
\end{abstract}

\section{INTRODUCTION}

Consider a Lévy process $\left(X_{t}, t \geq 0\right)$ started at $x \in[0, b]$ for some $b>0$ and let $Y_{t}$ be its reflection in the strip $[0, b]$ :

$$
Y_{t}=X_{t}+L_{t}-U_{t}
$$

where $Y_{t} \in[0, b], L_{0}=U_{0}=0$ and, moreover, $L, U$ are non-decreasing and their points of increase are contained in $\left\{t \geq 0: Y_{t}=0\right\}$ and $\left\{t \geq 0: Y_{t}=b\right\}$ respectively (this is a so-called two-sided Skorokhod problem). In the context of insurance risk theory, $X_{t}$ can be seen as a model for the surplus process of an insurance portfolio, and then $U_{t}$ and $L_{t}$ have the interpretation of the cumulative dividend payments (according to a horizontal barrier strategy at $b$ ) and injected capital, respectively (see e.g. Dickson and Waters [4]). In the queueing context, the respective quantities are the amount of work lost (in a queue with buffer $b$ ) and unused capacity, see e.g. Asmussen [2].

In this paper we will use the terminology of risk theory. Then the expected discounted dividends and expected discounted capital injections

$$
V_{U}^{b}(x)=\mathbb{E}_{x} \int_{0}^{\infty} e^{-\delta t} \mathrm{~d} U_{t}, \quad V_{L}^{b}(x)=\mathbb{E}_{x} \int_{0}^{\infty} e^{-\delta t} \mathrm{~d} L_{t}
$$

2010 Mathematics Subject Classification. Primary 60G51; Secondary 91B30.

Key words and phrases. Lévy processes, two-sided reflection, dividend strategies.

Financial support by the Swiss National Science Foundation Project 200020 143889 is gratefully acknowledged. 
are of particular interest, as they often serve as objectives for optimal control strategies. Here $x \in[0, b]$ is the initial surplus in the insurance portfolio and $\delta>0$ is the discount rate.

The aim of this paper is to establish simple identities between these and more general quantities using probabilistic techniques, which extends the set of respective explicit formulas available in the literature. In Section 2 we present a general sample path identity that links injections with dividend payments of the time-reversed path. When $X$ is a Lévy process, this duality leads to a direct link between the quantities in (2). In Section 3 we provide an alternative view on some of the known results concerning $V_{L}^{b}(x)$ and $V_{U}^{b}(x)$ for a spectrally-negative process. Finally, in Section 4 we obtain explicit expressions for expected discounted capital injections and dividend payments for a two-barrier strategy which Schmidli [10, 11] recently identified as optimal in various Lévy models when dividend payments are subject to taxation, but previous capital injections can be deducted from the dividends when paying taxes. Once again our focus is on a direct probabilistic proof.

\section{A SAMPLE PATH IDENTITY}

Let us start with stating an important alternative representation for the quantities in (2):

$$
V_{U}^{b}(x)=\mathbb{E}_{x} \int_{0}^{e_{\delta}} \mathrm{d} U_{t}=\mathbb{E}_{x} U_{e_{\delta}}, \quad V_{L}^{b}(x)=\mathbb{E}_{x} L_{e_{\delta}},
$$

where $e_{\delta}$ is an exponential random variable of rate $\delta$ independent of everything else. In this respect, observe that $X$ does not jump at $e_{\delta}$ a.s. since the set of jump times is countable. Note that $U_{e_{\delta}}$ and $\int_{0}^{\infty} e^{-\delta t} \mathrm{~d} U_{t}$ are two different random variables, but they have a common mean (and analogously for $L$ ), and it is the mean that is our focus. Throughout this paper we assume that $\mathbb{E}_{0} X_{1}$ is well defined and finite, so that both $V_{U}^{b}$ and $V_{L}^{b}$ are finite.

An easy consequence of (1) and (3) is the following bound on the difference of discounted expected dividends and injections:

$$
V_{U}^{b}(x)-V_{L}^{b}(x)=\left[\frac{\mathbb{E}_{0} X_{1}}{\delta}+x-b, \frac{\mathbb{E}_{0} X_{1}}{\delta}+x\right] \quad \forall b>0,
$$

because $\mathbb{E}_{0} X_{e_{\delta}}=\mathbb{E}_{0} X_{1} / \delta$. More interestingly, there is an identity relating dividends and injections, but the initial capital has to be chosen differently - it is at 0 for injections and at $b$ for dividends:

$$
V_{U}^{b}(b)-V_{L}^{b}(0)=\mathbb{E}_{0} X_{1} / \delta .
$$

The proof is based on a sample path identity that is interesting in its own right: Consider a sample path $X_{t}$ (which in fact can be any càdlàg 
function) and its time-reversed path defined for a fixed $T>0$ by

$$
\widehat{X}_{t}=X_{T}-X_{(T-t)-}, \quad t \in[0, T],
$$

where $X_{0-}=0$. It is well known that if $X$ is a Lévy process then $\widehat{X}$ has the same law as $X$ on $[0, T]$, see e.g. [7, Lem. 3.4]. We now provide an intriguing path-wise identity linking total dividends and capital injections:

Proposition 1. For any $b>0$ and every sample path it holds that

$$
\widehat{U}_{T}(b)-L_{T}(0)=X_{T},
$$

where $L_{T}(0)$ represents the total capital injections up to time $T$ corresponding to $X$ started at 0 , and $\widehat{U}_{T}(b)$ is the total dividends up to time $T$ corresponding to the time-reversed $\widehat{X}$ started at $b$, and both processes are reflected to stay in $[0, b]$.

Before we provide a proof let us consider two boundary cases. Firstly, if $b=\infty$ then $\widehat{U}_{T}(\infty)=\widehat{\widehat{X}}_{T}=X_{T}-\underline{X}_{T}$ and $L_{T}(0)=-\underline{X}_{T}$, and so the result is obvious. Secondly, if $X$ has bounded variation on $[0, T]$ then it makes sense to let $b \downarrow 0$. In this case we obtain $\widehat{U}_{T}(0)=\widehat{X}_{T}^{\uparrow}=X_{T}^{\uparrow}$ and $L_{T}(0)=-X_{T}^{\downarrow}$, where $X^{\downarrow}$ and $X^{\uparrow}$ measure the total decrease and total increase of $X$. Again the result is then obvious.

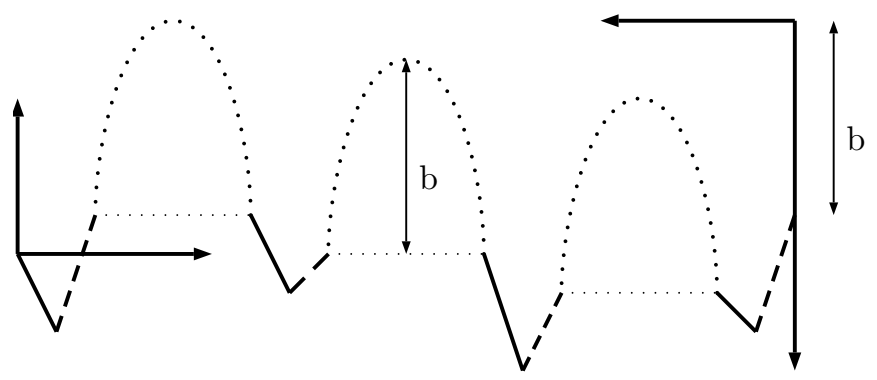

Figure 1. Schematic sample path: capital injections for the original and dividend payments for time-reversed process.

Proof of Proposition 1. For a general $b>0$ the identity of interest can be easily understood by inspecting Figure 1. The left and right axes correspond to the original and time-reversed sample paths, respectively. The solid segments depict capital injections in the original model, whereas dashed segments depict dividends for the time-reversed sample path. Thus we are interested in the total height of dashed segments minus the total height of solid segments, which must result in $X_{T}$. 
Remark 1. Similarly to Proposition 1 one may try to relate $L_{T}(0)$ and $U_{T}(0)$. Indeed, their difference is also 0 inside each excursion from the running minimum with height exceeding $b$. However, here the last (incomplete) excursion is more complex, not allowing to formulate an analogous simple relation.

It should be mentioned that Proposition 1 can be seen as a counterpart of Lindley's duality [8], where the event $\left\{Y_{T} \geq x\right\}$ is related to the two-sided exit problem of $\widehat{X}$ from $[x-b, x)$, see [2, Prop. XIV.3.7] and a finite horizon version in [6, Lem. 2].

\section{Spectrally NEGAtive LÉvy PROCESSES}

In the rest of the paper we assume that $X$ is spectrally negative, i.e. it can only have negative jumps, and it is not a non-increasing process. Let $\psi(\theta)=\log \mathbb{E} e^{\theta X_{1}}$ be its Laplace exponent, which is finite for all $\theta \geq 0$. Let $\Phi_{\delta}$ be the unique positive solution of $\psi(\cdot)=\delta$, and let $W_{\delta}(x), x \geq 0$ be the scale function. That is, $W_{\delta}$ is a continuous non-negative function characterized by its transform

$$
\int_{0}^{\infty} e^{-\theta x} W_{\delta}(x) \mathrm{d} x=1 /(\psi(\theta)-\delta)
$$

for $\theta>\Phi_{\delta}$. Furthermore, define the so-called second scale function by

$$
Z_{\delta}(x):=1+\delta \int_{0}^{x} W_{\delta}(y) \mathrm{d} y, \quad x \geq 0,
$$

and observe that $Z_{\delta}^{\prime}(x)=\delta W_{\delta}(x)$ and $Z_{\delta}(0)=1$, see e.g. Kyprianou 7]. Letting

$$
\tau_{y}^{ \pm}=\inf \left\{t \geq 0: \pm X_{t}> \pm y\right\}, \quad \rho_{y}:=\inf \left\{t \geq 0: Y_{t}>y\right\},
$$

we recall the basic exit formulas [7, §8] for $0 \leq x \leq y$ and $y>0$ :

$$
\begin{aligned}
\mathbb{E}_{x} e^{-\delta \tau_{y}^{+}} & =\frac{e^{\Phi_{\delta} x}}{e^{\Phi_{\delta} y}}=e^{-\Phi_{\delta}(y-x)}, \\
\mathbb{E}_{x}\left(e^{-\delta \tau_{y}^{+}} ; \tau_{y}^{+}<\tau_{0}^{-}\right) & =\frac{W_{\delta}(x)}{W_{\delta}(y)}
\end{aligned}
$$

as well as

$$
\mathbb{E}_{x} e^{-\delta \rho_{y}}=\frac{Z_{\delta}(x)}{Z_{\delta}(y)} \quad \text { for } y \leq b .
$$

The fact that all these quantities are ratios of certain functions is a consequence of the strong Markov property and the absence of positive jumps (see e.g. Gerber et al. [5] for an intuitive reasoning). Moreover, these expressions also lead to correspondingly simple expressions for the 
expected discounted dividends in the respective models with a dividend barrier at $b$ and initial capital $0 \leq x \leq b$ :

$$
\frac{e^{\Phi_{\delta} x}}{\Phi_{\delta} e^{\Phi_{\delta} b}}, \quad \frac{W_{\delta}(x)}{W_{\delta}^{\prime}(b)}, \quad \frac{Z_{\delta}(x)}{Z_{\delta}^{\prime}(b)}=: V_{U}^{b}(x) .
$$

In the first model the process is allowed to be negative (the event of ruin), in the second it is terminated upon becoming negative, and in the third capital is injected whenever the surplus is negative.

Let us briefly discuss two ways to derive the formulas in (7) using the model with injections as an illustration:

(i) The first argument is in the spirit of [5]: Observe that $V_{U}^{b^{\prime}}(b)=1$ which easily follows from the bound $h \mathbb{E}_{0} e^{-\delta \tau_{h}^{+}} \leq V_{U}^{b}(b)-V_{U}^{b}(b-h) \leq h$ and the fact that $\tau_{h}^{+} \downarrow 0$ as $h \downarrow 0 \mathbb{P}_{0}$-a.s. But $V_{U}^{\bar{b}}(x)=Z_{\delta}(x) / Z_{\delta}(b) V_{U}^{\bar{b}}(b)$ which yields $V_{U}^{b}(b)=Z_{\delta}(b) / Z_{\delta}^{\prime}(b)$ and the formula for $V_{U}^{b}(x)$.

(ii) When $x=b, U_{e_{\delta}}$ is an exponential random variable (of rate $\lambda_{b}$, say). Hence $V_{U}^{b}(b)=1 / \lambda_{b}$. But (6) shows that $Z_{\delta}(b+h) / Z_{\delta}(b) \leq$ $e^{-\lambda_{b} h} \leq Z_{\delta}(b) / Z_{\delta}(b-h)$ and then $\lambda_{b}=Z_{\delta}^{\prime}(b) / Z_{\delta}(b)$ follows, see also [1].

Remark 2. It may be interesting to note that $\lambda_{b}=\left(\log Z_{\delta}(b)\right)^{\prime}$, i.e. it is a logarithmic derivative of $Z_{\delta}(b)$. This can be explained by the identity $\mathbb{E}_{0} e^{-\delta \rho_{b}}=1 / Z_{\delta}(b)=\exp \left(-\int_{0}^{b} \lambda_{x} \mathrm{~d} x\right)$. That is, we may regard $\lambda_{x}$ as the rate of an inhomogeneous Poisson process describing killing of the first passage process $\rho_{x}, x \in[0, b]$.

Now as a consequence of (4) and (7) we get

$$
V_{L}^{b}(0)=\frac{Z_{\delta}(b)}{Z_{\delta}^{\prime}(b)}-\frac{\psi^{\prime}(0)}{\delta}, \quad V_{L}^{\infty}(0)=\frac{1}{\Phi_{\delta}}-\frac{\psi^{\prime}(0)}{\delta},
$$

because $1 / \Phi_{\delta}$ is the expected sum of discounted dividends in the model without ruin nor capital injections (cf. the first expression in (7)). Moreover, we have for $0<a \leq b$ :

$$
\Delta(a, b)=V_{L}^{a}(0)-V_{L}^{b}(0)=V_{U}^{a}(a)-V_{U}^{b}(b)=\frac{Z_{\delta}(a)}{Z_{\delta}^{\prime}(a)}-\frac{Z_{\delta}(b)}{Z_{\delta}^{\prime}(b)} .
$$

Furthermore, for any $x \leq a$, neither of the barriers $a$ or $b$ is met before $x$ is hit by the process started at 0 and reflected to stay positive, and so we get

$$
V_{L}^{a}(x)-V_{L}^{b}(x)=Z_{\delta}(x) \Delta(a, b) .
$$

In particular, for $b \rightarrow \infty$ this leads in a simple way to the identity

$$
V_{L}^{a}(x)=Z_{\delta}(x)\left(\frac{Z_{\delta}(a)}{Z_{\delta}^{\prime}(a)}-\frac{1}{\Phi_{\delta}}\right)+V_{L}^{\infty}(x) .
$$

Note that in Theorem 1 of Avram et al. 3] it was shown that

$$
V_{L}^{a}(x)=Z_{\delta}(x) \frac{Z_{\delta}(a)}{Z_{\delta}^{\prime}(a)}-\int_{0}^{x} Z_{\delta}(y) \mathrm{d} y-\frac{\psi^{\prime}(0)}{\delta},
$$


which is indeed consistent with (9). We will, however, not rely on this particular expression in the following.

Finally, let $\tau_{0}^{b}$ be the first passage time below 0 of the process reflected at the upper barrier $b$. Then we must have

$$
V_{U}^{b}(x)=\mathbb{E}_{x} e^{-\delta \tau_{0}^{b}} V_{U}^{b}(0)+\frac{W_{\delta}(x)}{W_{\delta}^{\prime}(b)},
$$

where the last term corresponds to the expected discounted dividends up to $\tau_{0}^{b}$, i.e. up to the classical ruin time. Thus from (7) we immediately obtain the following well known formula [9]

$$
\mathbb{E}_{x} e^{-\delta \tau_{0}^{b}}=Z_{\delta}(x)-Z_{\delta}^{\prime}(b) \frac{W_{\delta}(x)}{W_{\delta}^{\prime}(b)}
$$

\section{A MODEL WITH TWO DIVIDEND BARRIERS}

In this section we consider the model introduced by Schmidli [10, 11] in the context of optimal capital injection and dividend payout strategies, where dividends are subject to tax (with a constant rate $\gamma \in(0,1))$ whenever their total amount exceeds the total previously injected capital. The objective was to maximize expected discounted dividends after tax minus expected discounted capital injections (where the latter are penalized by a factor $\eta>1$ ). In the cases where $X$ is a classical risk process or its diffusion approximation the following model was found to be optimal:

There are two dividend barriers $a$ and $b>a$, and one capital injection barrier at 0 . The barrier $a$ is active whenever there are still capital injections to be amortized (so that the dividends paid at barrier $a$ are not taxed), and when there are no more amortizations, the barrier at $a$ is dissolved and a barrier strategy at $b$ becomes active, until new capital injections are needed and barrier $a$ is again installed, and so on. In Figure 2 we present a graphical illustration of this strategy, where the times $T_{i}$ correspond to dissolving the barrier at $a$.

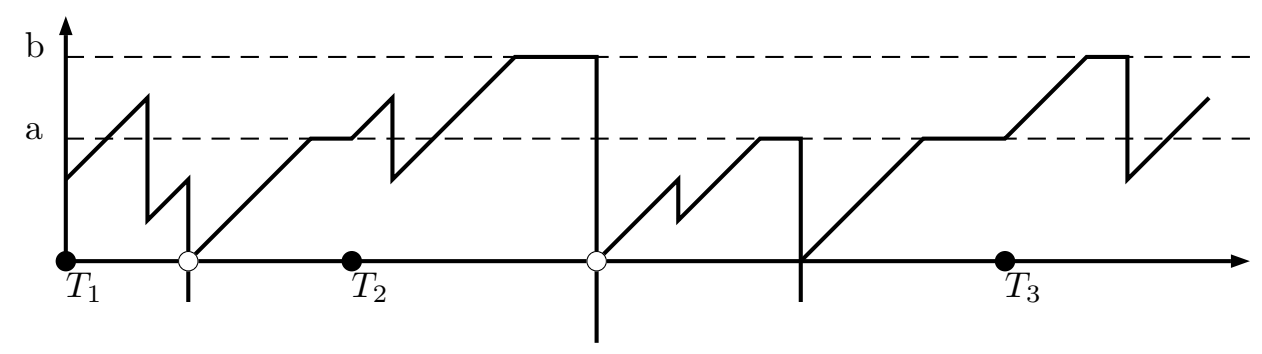

Figure 2. Schematic sample path: two dividend barriers. 
Our aim is to identify in a simple probabilistic way the expected discounted dividends $V_{U}^{a, b}(x, \ell)$ paid at ("upper") barrier $b$, the expected discounted dividends $V_{I}^{a, b}(x, \ell)$ paid at ("intermediate") barrier $a$ and the expected discounted capital injections $V_{L}^{a, b}(x, \ell)$ at ("lower") barrier 0 , where we also allow for some initial tax exemption $\ell \geq 0$ (think of $\ell$ as capital injections before time 0 ). In particular, the above mentioned objective for the initial capital $x \in[0, b]$ is to maximize

$$
(1-\gamma) V_{U}^{a, b}(x, 0)+V_{I}^{a, b}(x, 0)-\eta V_{L}^{a, b}(x, 0) .
$$

The following result establishes a link between the three quantities of interest, additionally using the classical quantities $V_{U}^{b}(x)$ and $V_{L}^{b}(x)$ corresponding to the two-sided reflection in $[0, b]$ (that is, no barrier at $a$ ). The main idea is to identify certain invariance properties by considering ratios and differences of some value functions.

Proposition 2. For any initial capital $x \in[0, a]$, initial tax exemption $\ell \geq 0$ and discount rate $\delta>0$ it holds that

$$
V_{U}^{a, b}(x, \ell)=\frac{1}{\Phi_{\delta}} e^{-\Phi_{\delta}(b+\ell-x)},
$$

$$
V_{I}^{a, b}(x, \ell)=\left(V_{U}^{b}(x)-V_{U}^{a, b}(x, \ell)\right) \frac{W_{\delta}(b)}{W_{\delta}(a)},
$$

$$
V_{L}^{a, b}(x, \ell)=V_{L}^{b}(x)+\left(V_{U}^{b}(x)-V_{U}^{a, b}(x, \ell)\right)\left(\frac{W_{\delta}(b)}{W_{\delta}(a)} Z_{\delta}(a)-Z_{\delta}(b)\right) .
$$

The above formulas also hold for $x \in(a, b]$, when $\ell=0$.

Proof. Since any capital injection is taken out of the process as dividends again at level $a, X$ always reaches the barrier $b>a$ at the times exactly as if there were no injections and no ruin, and so formula (11) immediately follows from (7).

Part I: Assume $x \in[0, a]$.

To establish (12), we proceed as follows. Using the third formula in (7) we find that

$$
V_{U}^{a}(x) / V_{U}^{b}(x)=W_{\delta}(b) / W_{\delta}(a),
$$

where the right hand side does not depend on the common initial level $x$. Recall that $\tau_{0}^{b}$ is the ruin time of the process with dividend barrier $b$ and no injections. Let further $v_{U}^{a}(x, b)$ be the expected discounted dividends up to $\tau_{0}^{b}$ of the process starting at $x$ with horizontal barrier at $a$ in the presence of injections. Clearly

$$
V_{U}^{a}(x)=v_{U}^{a}(x, b)+\mathbb{E}_{x} e^{-\delta \tau_{0}^{b}} V_{U}^{a}(0),
$$


because $a \leq b$. Since trivially also

$$
V_{U}^{b}(x)=v_{U}^{b}(x, b)+\mathbb{E}_{x} e^{-\delta \tau_{0}^{b}} V_{U}^{b}(0),
$$

and using (14), we find that

$$
v_{U}^{a}(x, b) / v_{U}^{b}(x, b)=W_{\delta}(b) / W_{\delta}(a) .
$$

Importantly, we have the following representation of the difference: for $\ell>0$

$$
V_{U}^{a}(x)-V_{I}^{a, b}(x, \ell)=\mathbb{E}_{x} e^{-\delta T_{1}} v_{U}^{a}(a, b)+\sum_{n=2}^{\infty} \mathbb{E}_{x} e^{-\delta T_{n}} v_{U}^{a}(a, b),
$$

where $T_{n}$ is the time in the two-dividend barrier model when the process stops being reflected at $a$ for the $n$th time. Note that $T_{n}$ depends on $x$ and $\ell$. For $\ell=0$, we take $T_{1}=0$ (see Figure 2) and the above equation is

$$
V_{U}^{a}(x)-V_{I}^{a, b}(x, 0)=\mathbb{E}_{x} v_{U}^{a}(x, b)+\sum_{n=2}^{\infty} \mathbb{E}_{x} e^{-\delta T_{n}} v_{U}^{a}(a, b) .
$$

For both cases, replacing $v_{U}^{a}(\cdot, b)$ by $v_{U}^{b}(\cdot, b) W_{\delta}(b) / W_{\delta}(a)$ we readily obtain

$$
V_{U}^{a}(x)-V_{I}^{a, b}(x, \ell)=V_{U}^{a, b}(x, \ell) \frac{W_{\delta}(b)}{W_{\delta}(a)},
$$

which by virtue of 14 immediately yields 12 .

Next, we prove $(13)$. Consider the difference of expected discounted injections in (8), and let $\Delta_{L}^{a, b}(x)$ be this difference considered up to the time $\tau_{0}^{b}$ which according to 10 must be

$$
\begin{aligned}
\Delta_{L}^{a, b}(x) & =\left(V_{L}^{a}(x)-V_{L}^{b}(x)\right)-\mathbb{E}_{x} e^{-\delta \tau_{0}^{b}}\left(V_{L}^{a}(0)-V_{L}^{b}(0)\right) \\
& =\Delta(a, b) Z_{\delta}^{\prime}(b) \frac{W_{\delta}(x)}{W_{\delta}^{\prime}(b)}=\Delta(a, b) Z_{\delta}^{\prime}(b) v_{U}^{b}(x, b) .
\end{aligned}
$$

Similarly to the above we have

$$
\begin{aligned}
V_{L}^{a}(x)-V_{L}^{a, b}(x, \ell) & =\mathbb{E}_{x} e^{-\delta T_{1}} \Delta_{L}^{a, b}(a)+\sum_{n=2}^{\infty} \mathbb{E}_{x} e^{-\delta T_{n}} \Delta_{L}^{a, b}(a) \\
& =V_{U}^{a, b}(x, \ell) \Delta(a, b) Z_{\delta}^{\prime}(b)
\end{aligned}
$$

(for $\ell=0$ again replace the left $\Delta_{L}^{a, b}(a)$ by $\Delta_{L}^{a, b}(x)$ in the first line, which leads to the same second line also for that case). This already gives a nice expression for $V_{L}^{a, b}(x, \ell)$, which further can be converted using (8) to

$$
V_{L}^{a, b}(x, \ell)=V_{L}^{b}(x)+\left(\frac{Z_{\delta}(x)}{Z_{\delta}^{\prime}(b)}-V_{U}^{a, b}(x, \ell)\right) \Delta(a, b) Z_{\delta}^{\prime}(b),
$$

and (13) follows. 
Part II: Assume $x \in(a, b]$ and $\ell=0$.

For $x>a$ the basic formulas (8) and (14) do not hold any more, which necessitates to treat this case differently. In fact, our formulas generalize easily by conditioning on the process at the time $\tau_{0}^{b}$.

Considering (12) we may write

$$
\begin{aligned}
V_{I}^{a, b}(x, 0) & =\mathbb{E}_{x} e^{-\delta \tau_{0}^{b}} V_{I}^{a, b}\left(0, \ell\left(\tau_{0}^{b}\right)\right) \\
& =\mathbb{E}_{x} e^{-\delta \tau_{0}^{b}}\left(V_{U}^{b}(0)-V_{U}^{a, b}\left(0, \ell\left(\tau_{0}^{b}\right)\right)\right) \frac{W_{\delta}(b)}{W_{\delta}(a)} \\
& =\left(V_{U}^{b}(x)-V_{U}^{a, b}(x, 0)\right) \frac{W_{\delta}(b)}{W_{\delta}(a)},
\end{aligned}
$$

where $\ell\left(\tau_{0}^{b}\right)$ is the amount of injections added at $\tau_{0}^{b}$. Here we also used the fact that contributions to $V_{U}^{b}(x)$ and $V_{U}^{a, b}(x, 0)$ before $\tau_{0}^{b}$ will cancel out. Similarly with regard to (13) we obtain

$$
\begin{aligned}
V_{L}^{a, b}(x, 0) & =\mathbb{E}_{x} e^{-\delta \tau_{0}^{b}} V_{L}^{a, b}\left(0, \ell\left(\tau_{0}^{b}\right)\right)+\mathbb{E}_{x} e^{-\delta \tau_{0}^{b}} \ell\left(\tau_{0}^{b}\right) \\
& =\left(\mathbb{E}_{x} e^{-\delta \tau_{0}^{b}} V_{L}^{b}(0)+\mathbb{E}_{x} e^{\left.-\delta \tau_{0}^{b} \ell\left(\tau_{0}^{b}\right)\right)}\right. \\
& +\mathbb{E}_{x} e^{-\delta \tau_{0}^{b}}\left(V_{U}^{b}(0)-V_{U}^{a, b}\left(0, \ell\left(\tau_{0}^{b}\right)\right)\right)\left(\frac{W_{\delta}(b)}{W_{\delta}(a)} Z_{\delta}(a)-Z_{\delta}(b)\right) \\
& =V_{L}^{b}(x)+\left(V_{U}^{b}(x)-V_{U}^{a, b}(x, 0)\right)\left(\frac{W_{\delta}(b)}{W_{\delta}(a)} Z_{\delta}(a)-Z_{\delta}(b)\right)
\end{aligned}
$$

completing the proof.

\section{REFERENCES}

[1] H. Albrecher and J. Ivanovs. Power identities for Lévy risk models under taxation and capital injections. Stochastic Systems, 4(1):157-172, 2014.

[2] S. Asmussen. Applied probability and queues, volume 51 of Applications of Mathematics (New York). Springer-Verlag, New York, second edition, 2003. Stochastic Modelling and Applied Probability.

[3] F. Avram, Z. Palmowski, M. R. Pistorius, et al. On the optimal dividend problem for a spectrally negative Lévy process. The Annals of Applied Probability, 17(1):156-180, 2007.

[4] D. Dickson and H. Waters. Some optimal dividend problems. Astin Bull., 34(1):49-74, 2004.

[5] H. U. Gerber, X. S. Lin, and H. Yang. A note on the dividends-penalty identity and the optimal dividend barrier. Astin Bulletin, 36(02):489-503, 2006.

[6] J. Ivanovs. Potential measures of one-sided markov additive processes with reflecting and terminating barriers. Journal of Applied Probability, 51(4):1154$1170,2014$.

[7] A. E. Kyprianou. Introductory lectures on fluctuations of Lévy processes with applications. Springer Science \& Business Media, 2006.

[8] D. Lindley. Discussion on Mr. Winsten's paper. Journal of the Royal Statistical Society, B21(1):22-23, 1959.

[9] M. R. Pistorius. On exit and ergodicity of the spectrally one-sided Lévy process reflected at its infimum. J. Theoret. Probab., 17(1):183-220, 2004. 
[10] H. Schmidli. On capital injections and dividends with tax in a classical risk model. Insurance: Mathematics and Economics, 71:138-144, 2016.

[11] H. Schmidli. On capital injections and dividends with tax in a diffusion approximation. Scandinavian Actuarial Journal, in press, 2016.

E-mail address: hansjoerg.albrecher@unil.ch

University of Lausanne and Swiss Finance Institute, Quartier UNILDorigny, BÂtiment Extranef, 1015 Lausanne, Switzerland

E-mail address: jevgenijs.ivanovs@math.au.dk

Aarhus University, Ny Munkegade 118, DK-8000 Aarhus C, Denmark 